\title{
Circulating Tumor Cells as a Biomarker in Pancreatic Ductal Adenocarcinoma
}

\author{
Han Liu ${ }^{a}$ Bo Sun ${ }^{b}$ Shengnan Wanga Congjin Liu ${ }^{a}$ Yun Luª Ding Lia \\ Xingdang Liu ${ }^{a}$
}

${ }^{\mathrm{a}}$ Department of Nuclear Medicine, Huashan Hospital, Fudan University, Shanghai, b Department of General Surgery, Huashan Hospital, Fudan University, Shanghai, China

\section{Key Words}

Circulating tumor cells $•$ Pancreatic cancer $\bullet$ Aneuploidy $\bullet$ Biomarker

\begin{abstract}
Background/Aims: Circulating tumor cells (CTCs) are valuable in both basic research and clinical application for cancer management. In the current study, we evaluated the diagnostic value of CTCs in pancreatic ductal adenocarcinoma (PDAC). Methods: In total, 143 blood samples from 95 consecutively diagnosed PDAC patients and 48 healthy donors were collected. Combined data from immunostaining of CD45, DAPI and fluorescence in situ hybridization (FISH) with chromosome 8 centromere (CEP8) probe were used to identify CTCs. Cells with features of CD45-/DAPI+/CEP8>2 were detected as CTCs. Results: CTCs were classified as triploid, tetraploid and multiploid based on chromosome 8 copy number. CTC subtype composition was significantly different among groups. Both subtype number and total CTC number were significantly increased in PDAC patients, compared to healthy controls. Total CTC number had $75.8 \%$ sensitivity and $68.7 \%$ specificity at a cutoff value of 2 cells $/ 3.2 \mathrm{~mL}$. This study is the first to report that CTC subtype number is also useful in cancer diagnosis. Sensitivity was $53.7 \%$ and specificity was $85.4 \%$ at a cutoff point of 2 CTC subtypes. The diagnostic value of both total CTC number and CTC subtype number was a little poorer than CA199. Conclusions: Both CTC subtype and total CTC number may serve as potential biomarkers for PDAC.
\end{abstract}

(C) 2017 The Author(s)

Published by S. Karger AG, Basel

\section{Introduction}

Pancreatic ductal adenocarcinoma (PDAC), derived from the ductal epithelium, is the most common histological subtype accounting for $90 \%$ of all pancreatic malignancies [1]. Symptoms generally occur later on during disease progression, the most frequent being pain, jaundice and weight loss. The majority of PDAC patients have metastatic disease at the time of diagnosis. Diagnostic imaging tests, including CT and MRI, have the ability to depict the location, size and vascularity of pancreatic cancer, but show limited potential in detecting small primary tumors and metastatic locations [2]. Endoscopic ultrasound coupled H. Liu and B. Sun contributed equally to this work. 
with fine-needle aspiration (EUS-FNA) is the current gold standard technique [3]. However, this method requires sedation and is associated with appreciable risk of complications, such as pancreatitis, bowel perforation and aspiration. Different serum biomarkers are used as adjuncts in the diagnosis of PDAC, among which carbohydrate antigen 19-9 (CA199) is the only biomarker approved by the Food and Drug Administration (FDA) of the United States [4]. Although CA199 is widely adopted in the diagnosis and treatment process, it is insufficiently sensitive for early detection screening and non-specific due to elevated levels in a number of benign pancreatic or biliary diseases. The identification of effective biomarkers for early diagnosis and dynamic management of PDAC thus remains an urgent unmet medical need.

Circulating tumor cells (CTCs) are tiny amounts of tumor cells that have escaped from primary tumors and metastatic foci and entered the bloodstream [5, 6]. These cells have recently been exploited for guiding clinical management, evaluating curative efficacy, predicting prognosis and monitoring tumor recurrence [7]. The CellSearch system is the only CTC detection technology approved by the FDA. The identification procedure relies on cytokeratin staining of cells in blood samples [8]. However, the CellSearch system is limited, in that epithelial cell adhesion molecules and cytokeratins are not expressed in a number of CTCs. A number of novel methodologies have been developed to improve the efficiency and precision of CTC detection in pancreatic cancer [9]. Aneuploidy, a common manifestation of chromosome instability, is a hallmark of malignant solid tumors [10]. As chromosome numbers are reflected by chromosome 8 centromere (CEP8) using fluorescence in situ hybridization (FISH), aneuploidy detection in peripheral blood provides a fresh perspective for CTC detection [11].

In this study, a total of 143 blood samples from 95 consecutively diagnosed pancreatic ductal adenocarcinoma patients and 48 healthy donors were enrolled for CTC identification. Combined immunostaining of CD45, DAPI and CEP8-FISH was performed to detect and characterize CTCs. CTC subtype distribution and composition data from different groups were inputted for statistical analysis. We further explored the associations of total CTC number and CTC subtype number with clinicopathological factors. The receiver operating characteristics (ROC) curve was constructed to evaluate the diagnostic value of CTCs.

\section{Materials and Methods}

\section{Patients and sample collection}

Ninety-five patients with newly confirmed PDAC were enrolled from April 2015 to April 2016. None of the included patients had received any PDAC-directed treatment or had a history of any other malignancy during the past 5 years. Forty-eight healthy donors, randomly selected from physical examination center, were included as controls. Criteria for healthy donors included no family history of cancer, no tumors or other systemic disease history. All subjects signed an informed consent agreement. Diagnosis of PDAC was based on histopathological observations of specimens obtained from fine needle aspiration or resection. Clinical data, including age, gender, location of primary tumor, tumor differentiation, tumor size, lymph node metastasis and distant metastases, were collected. Clinical staging was based on the current classification system designed by American Joint Committee on Cancer (AJCC).

Whole peripheral blood samples were obtained via venipuncture from healthy donors and PDAC patients before invasive tests and therapeutic measures. Tubes without anti-coagulant were used to collect $2 \mathrm{~mL}$ blood samples from each subject for clinical tumor biomarker analysis. Tubes containing acid citrate dextrose (ACD)-anticoagulant (Becton Dickinson, NJ, USA) were used to obtain $3.2 \mathrm{~mL}$ whole blood samples for CTC detection. All blood samples were processed within $48 \mathrm{~h}$ after collection. The experimental protocol was approved by the Ethics Committee of Fudan University-Affiliated Huashan Hospital and conducted in adherence to the Declaration of Helsinki Principles.

Subtraction enrichment and detection of CTCS

Strategies for CTC isolation and detection were similar to those reported previously [12]. In summary, $4 \mathrm{~mL}$ collected blood (including $0.8 \mathrm{ml}$ anticoagulant) was centrifuged to separate cells from whole blood. 
Next, RBCs were dissolved via RBC hypotonic hemolysis, and residual cell particles resuspended in PBS. CTCs were cultured with anti-CD45 monoclonal antibody-coated magnetic beads (Life Technologies, Carlsbad, CA, USA). Subsequently, magnetic beads loaded with the majority of leukocytes were wiped out by a magnetic stand (Promega, Madison, WI, USA) from the cell suspension, which was smeared and fixed on slides for CTC identification. Specimens were dipped in $2 \times \mathrm{SSC}$ at $37^{\circ} \mathrm{C}$ for $15 \mathrm{~min}$, and dehydrated in $75 \%, 85 \%$ and $100 \%$ ethanol for $3 \mathrm{~min}$ each. Vysis Spectrum Orange-labeled centromere probe 8 (CEP8) was added (Abbott Molecular Diagnostics, Des Plaines, IL, USA). Specimens were denatured at $76^{\circ} \mathrm{C}$ for $5 \mathrm{~min}$ and hybridized at $37^{\circ} \mathrm{C}$ for $1.5 \mathrm{~h}$, followed by soaking in $50 \%$ formamide for $15 \mathrm{~min}$ and washing in $2 \times$ SSC twice for $5 \mathrm{~min}$. Specimens were dehydrated in a series of $75 \%, 85 \%$ and $100 \%$ ethanol for 3 min each, incubated with Alexa Fluor 594-conjugated anti-human CD45 at room temperature for $1 \mathrm{~h}$, and washed twice with $0.2 \%$ BSA. Following the addition of DAPI, slides were observed along the "S" track using a microscope (Nikon). CTCs were stained as CD45-/ DAPI+/CEP8>2. Enrichment and identification of CTCs were performed blindly by different personnel, who were not provided any information on the medical history and clinical status of patients.

Measurement of CA199

The expression levels of CA199 in serum samples were determined using a chemiluminescence assay kit according to the kit instructions of manufacturer (Roche, Shanghai, China). The cutoff value for the biomarker was $35 \mathrm{U} / \mathrm{mL}$.

\section{Statistical analysis}

Statistical analysis was carried out using SPSS Statistics 22.0 (SPSS, Chicago, IL, USA). ROC curve was plotted to assess the performance of CTCs in a PDAC screening test. Correlations of PDAC CTCs with clinicopathological characteristics were examined using the chi-square test. All statistical tests were twosided. $P$ values less than 0.05 were considered significant.

\section{Results}

\section{CTC identification}

CD45, DAPI and CEP8 staining data observed with the fluorescence microscope were combined to identify CTCs (Fig. 1). Immunostaining of CD45 was applied to exclude the disturbance of leukocytes from tumor cells derived from the pancreatic ductal epithelium. Cell nuclei were observed using DAPI stain. CEP8 was used to count chromosome 8 copy number. Theoretically, the size and strength of CEP8 signals in the nucleus of the same specimen should be consistent. Close hybridization signals in single cells were referred to as 1 . Small-scale and weak hybridization signals may have been non-specific and were thus not counted. Cells with features of CD45+/DAPI+/CEP8 $\leq 2$ (Fig. 1a), CD45+/DAPI+/CEP $>2$ (Fig. 1b) and CD45-/DAPI+/CEP8 $\leq 2$ (Fig. 1c) were considered negative, while those stained as CD45-/DAPI+/CEP>2 were defined as CTCs. Cells were classified as triploid, tetraploid, pentaploid, sextuploid and septuploid CTCs based on chromosome 8 copy number. As CTCs with more than 5 CEP8 signals were rarely seen in the study, they were collectively called as multiploid CTCs for later convenient searching. Cells were thus classified as triploid (Fig. 1d), tetraploid (Fig. 1e) and multiploid CTCs (Fig. 1f) based on chromosome 8 copy number.

\section{CTC subtype distribution and subtype composition in different groups}

In total, 448 CTCs were identified in 83 out of 95 PDAC patients, including 306 (68.3\%) triploid cells, $119(26.6 \%)$ tetraploid cells and 23 (5.1\%) multiploid cells. Within the 48 healthy controls, 37 (69.8\%) triploid cells, 13 (24.5\%) tetraploid cells and $3(5.6 \%)$ multiploid cells were detected. The result demonstrates that CTC identified by the number of CEP8 signals not only exist in peripheral blood of PDAC patients but also in the peripheral blood of healthy controls. This is in accordance with previous study, showing that aneuploidies exist in a small fraction in normal tissues [13]. The reported occurrence varies from $0.5 \%$ to $20 \%$, and appears different in different tissue. Aneuploidy has been reported to occur in 
Fig. 1. Identification CTCs. CD45+ (a, b) and CEP $8 \leq 2$ (c) cells were determined as CTC-negative. Hyperdiploid cells with no CD45-positive signals in peripheral blood samples were identified as circulating tumor cells (CTCs). Triploid CTC (d), tetraploid CTC (e) and pentaploid CTC (f).
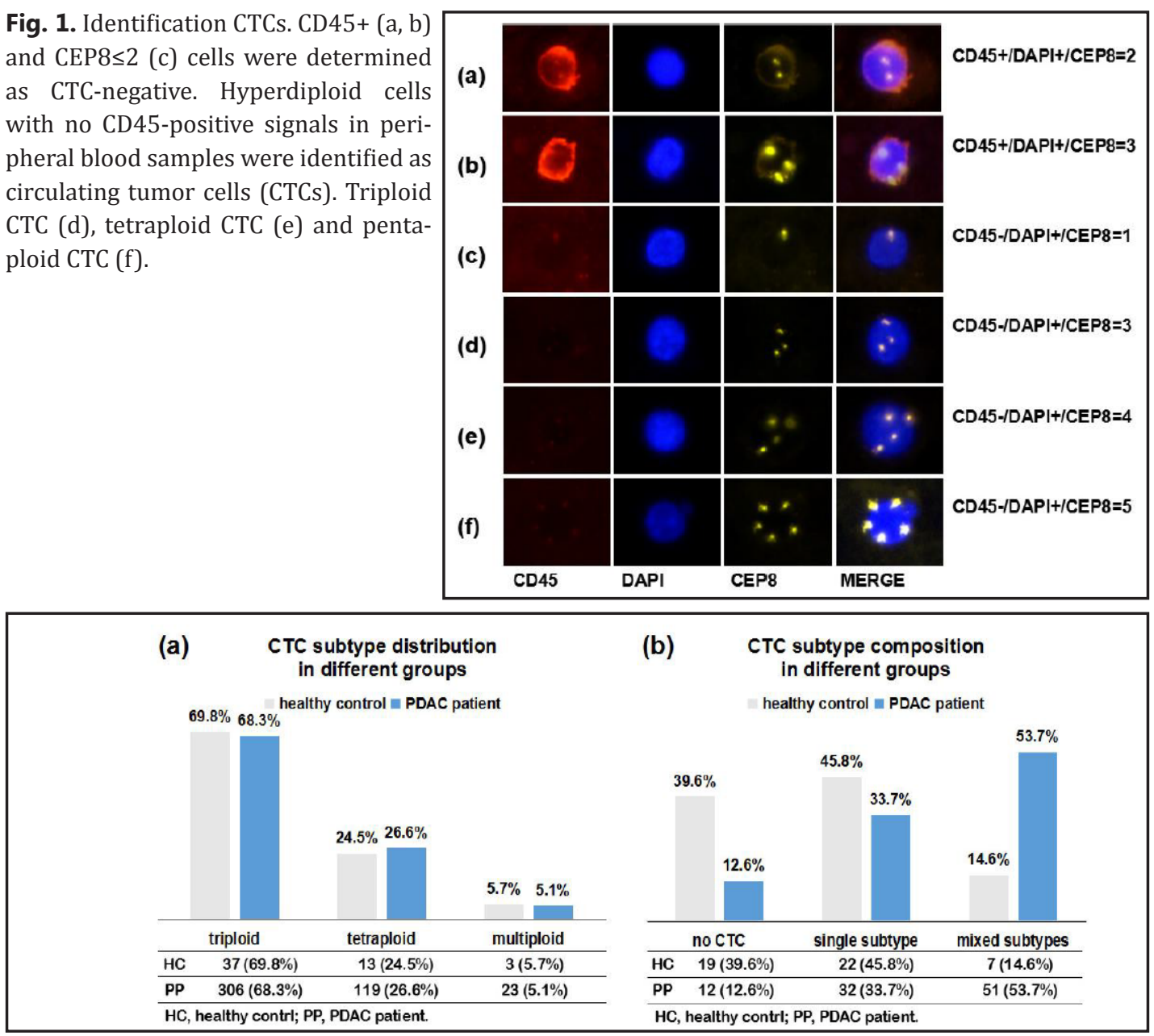

Fig. 2. (a) CTC subtype distribution in different groups. No significant differences in distribution of the three CTC subtypes were observed between PDAC patient and healthy control groups; (b) CTC subtype composition in different groups. CTC subtype composition was distinct for subjects from different groups.

Table 1. CTC subtype composition in PDAC group and healthy control group. Tri, triploid CTC; Tetra, tetraploid CTC; Multi, multiploid CTC

\begin{tabular}{lll}
\hline Group & PDAC patient & Healthy control \\
\hline no CTC & $12(12.6 \%)$ & $19(39.6 \%)$ \\
Tri & $29(30.5 \%)$ & $16(33.3 \%)$ \\
Tetra & $3(3.2 \%)$ & $5(10.4 \%)$ \\
Multi & $0(0.0 \%)$ & $1(2.1 \%)$ \\
Tri \& Tetra & $29(30.5 \%)$ & $5(10.4 \%)$ \\
Tri \& Multi & $6(6.3 \%)$ & $1(2.1 \%)$ \\
Tetra \& Multi & $3(3.2 \%)$ & $1(2.1 \%)$ \\
all subtypes & $13(13.7 \%)$ & $0(0.0 \%)$ \\
Summation & $95(100 \%)$ & $48(100 \%)$ \\
\hline
\end{tabular}

association with cellular stressors, such as oxidative damage, hypertension and ageing [14]. The occurrence of aneuploidies in the peripheral blood of healthy donors may shed from tissues under these cellular stressors. In PDAC patients, the more frequent occurrence of aneuploidies may arise from PDACs.

We compared the distribution of the three CTC subtypes between the PDAC and healthy control group and found no differences ( $p=0.944)$ (Fig. 2a). We then analyzed the CTC subtype composition of each subject from the patient and healthy control groups (Table 1). All three CTC subtypes were present in 13 out of $95(13.7 \%)$ patients and none of the healthy controls. 
(a)

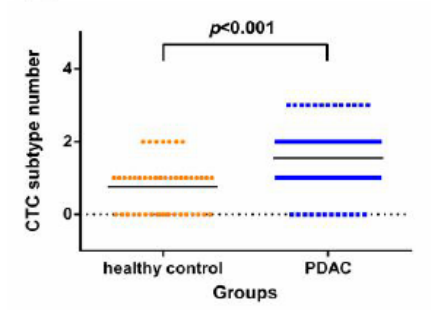

(b)

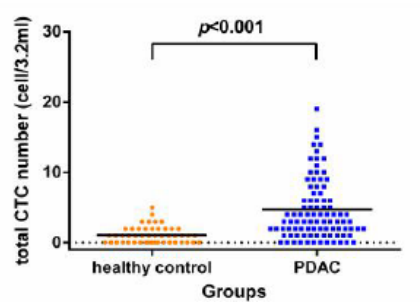

(c)

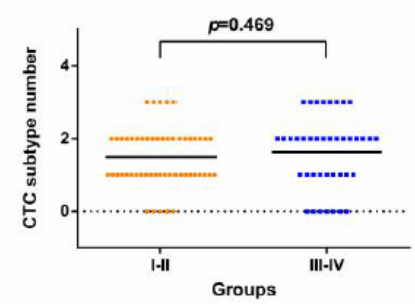

(d)

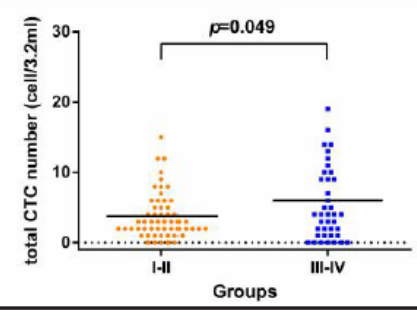

(e)

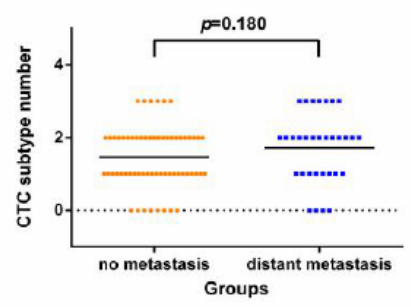

(f)

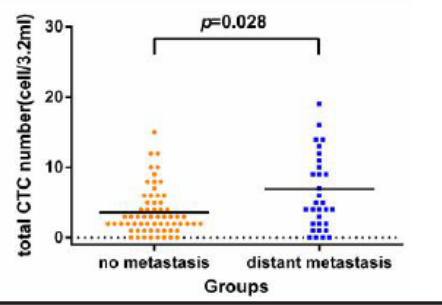

Fig. 3. CTC subtype number and total CTC number in pancreatic cancer patients and healthy controls. CTC subtype number and total CTC number in PDAC patients were significantly higher than those in healthy controls (a, b). CTC subtype number was not markedly different between stage I-II and stage III-IV patients (c). Total CTC number in patients with stage I-II tumors was significantly lower compared to that in stage III-IV (d). No significant differences in CTC subtype number were evident between patients with and without distant metastasis (e). Total CTC number in patients with distant metastasis was significantly increased relative to those with no distant metastasis (f).

(a)

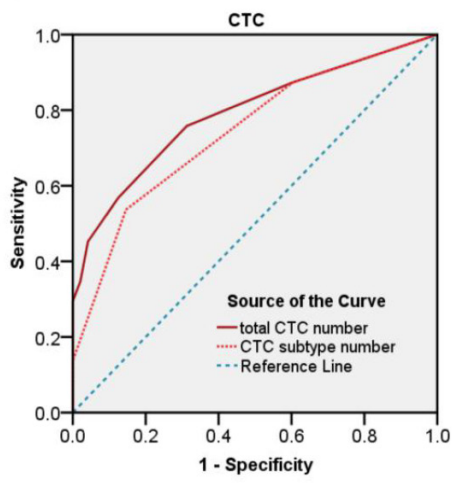

(b)

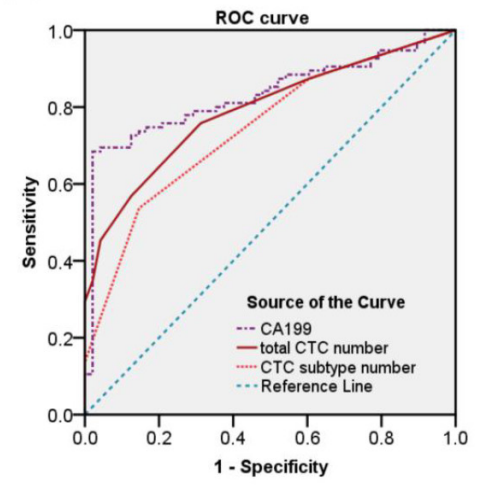

Fig. 4. (a) Value of area under the ROC curve for total CTC number and CTC subtype number. The area under the ROC curve was up to $0.791(95 \% \mathrm{CI}=0.718-0.863, \mathrm{p}<0.001)$ for total CTC number and $0.743(95 \% \mathrm{CI}=$ $0.661-0.825, \mathrm{p}<0.001$ ) for CTC subtype number. The optimal cutoff value was $2 \mathrm{CTCs} / 3.2 \mathrm{~mL}$ blood for total CTC number, yielding a sensitivity of $75.8 \%$ and specificity of $68.7 \%$. For CTC subtype number, sensitivity was $53.7 \%$ and specificity was $85.4 \%$ using 2 CTC subtypes as a cutoff point. (b) Value of the area under the ROC curve for CA199. Both area under the ROC curve for CTC subtype number and total CTC number were a little lower than CA199.

Both triploidy and tetraploidy were observed in 29 out of 95 (30.5\%) patients, but only 5 out of $48(10.4 \%)$ healthy controls. To determine whether CTC subtype composition of subjects differed between the patient and healthy control groups, we classified subtype composition of each subject into three categories: no CTC, single subtype and mixed subtypes with two or three subtypes (Fig. 2b). Notably, significant differences in CTC subtype composition were observed for subjects in the different groups $(p<0.01)$. Subjects from the PDAC patient group tended to display mixed CTC subtypes. 
Table 2. The relationship between CTC-positive rate and clinicopathological characteristics. No-neg, number-negative; No-posi, number-positive; Subneg, subtype-negative; Sub-posi, subtype-positive

\begin{tabular}{|c|c|c|c|c|c|c|c|}
\hline \multirow{2}{*}{ Variations } & \multirow{2}{*}{ Number } & \multicolumn{2}{|c|}{ No of patients } & \multirow[b]{2}{*}{$p$} & \multicolumn{2}{|c|}{ No of patients } & \multirow[b]{2}{*}{$p$} \\
\hline & & No-neg & No-posi & & Sub-neg & Sub-posi & \\
\hline \multicolumn{8}{|l|}{ Gender } \\
\hline Female & 41 & 9 & 32 & 0.654 & 20 & 21 & 0.675 \\
\hline Male & 54 & 14 & 40 & & 24 & 30 & \\
\hline \multicolumn{8}{|l|}{ Age } \\
\hline$\leq 60$ & 30 & 4 & 26 & 0.093 & 8 & 22 & 0.009 \\
\hline$>60$ & 65 & 19 & 46 & & 36 & 29 & \\
\hline \multicolumn{8}{|l|}{ Tumor location } \\
\hline Head or neck & 66 & 18 & 48 & 0.293 & 35 & 31 & 0.048 \\
\hline Body or tail & 29 & 5 & 24 & & 9 & 20 & \\
\hline \multicolumn{8}{|l|}{ Differentiation } \\
\hline Well or moderately & 43 & 8 & 35 & 0.311 & 20 & 23 & 0.530 \\
\hline poor & 20 & 6 & 14 & & 11 & 9 & \\
\hline uncertain & 32 & 9 & 23 & & 13 & 19 & \\
\hline \multicolumn{8}{|l|}{ Tumor stage } \\
\hline I-II & 55 & 12 & 43 & 0.523 & 28 & 27 & 0.292 \\
\hline III-IV & 40 & 11 & 29 & & 16 & 24 & \\
\hline \multicolumn{8}{|l|}{ Tumor size } \\
\hline T1-T2 & 25 & 9 & 16 & 0.109 & 15 & 10 & 0.110 \\
\hline T3-T4 & 70 & 14 & 56 & & 29 & 41 & \\
\hline \multicolumn{8}{|l|}{ Lymph nodes } \\
\hline No & 35 & 6 & 29 & 0.242 & 17 & 18 & 0.899 \\
\hline N1 & 46 & 13 & 33 & & 23 & 23 & \\
\hline uncertain & 14 & 4 & 10 & & 4 & 10 & \\
\hline \multicolumn{8}{|l|}{ Metastasis } \\
\hline Mo & 63 & 16 & 47 & 0.705 & 32 & 31 & 0.219 \\
\hline M1 & 32 & 7 & 25 & & 12 & 20 & \\
\hline \multicolumn{8}{|l|}{ CA19-9 } \\
\hline$<37 \mathrm{U} / \mathrm{ml}$ & 29 & 6 & 23 & 0.595 & 13 & 16 & 0.847 \\
\hline$\geq 37 \mathrm{U} / \mathrm{ml}$ & 66 & 17 & 49 & & 31 & 35 & \\
\hline
\end{tabular}

Table 3. Sensitivity and specificity comparison between CA199 and CTCs. AUC, area under the curve

\begin{tabular}{llllll}
\hline Method & Cutoff point & Sensitivity & Specificity & AUC & $p$ \\
\hline total CTC number & 2 cells/3.2mL & $75.8 \%$ & $68.7 \%$ & 0.791 & $<0.001$ \\
CTC subtype number & 2 subtypes $/ 3.2 \mathrm{~mL}$ & $53.7 \%$ & $85.4 \%$ & 0.743 & $<0.001$ \\
CA199 & $45.17 \mathrm{U} / \mathrm{mL}$ & $68.4 \%$ & $97.9 \%$ & 0.831 & $<0.001$ \\
\hline
\end{tabular}

CTC subtype number and total CTC number in pancreatic cancer patients and healthy controls

We further analyzed CTC subtype number and total CTC number in the different groups. The mean CTC subtype number detected in PDAC patients was $1.55(0-3)$, but just $0.75(0-2)$ in healthy controls. This difference was statistically significant $(p<0.001)$ (Fig. 3a). Overall, 0-31 total CTCs/3.2 mL whole blood (mean number, $4.71 \mathrm{CTCs} / 3.2 \mathrm{~mL}$ ) were detected in pancreatic cancer patients relative to $0-5$ total CTCs $/ 3.2 \mathrm{~mL}$ (median number, $1.10 \mathrm{CTCs} / 3.2$ $\mathrm{mL}$ ) in healthy controls, which was significantly different $(p<0.001)$ (Fig. $3 \mathrm{~b})$. As TNM staging is commonly applied in the diagnosis, cure and prognosis of PDAC, we examined the relationship between TNM stage and CTC. The mean CTC subtype number in patients with stage I-II tumors was $1.49(0-3)$ and that in patients with stage III-IV tumors was 1.63 $(0-3)$. There was no difference in CTC subtype number between patients with stage I-II and stage III-IV tumors ( $\mathrm{p}=0.469$ ) (Fig. 3c). The mean number of CTCs in patients with stage I-II tumors was $3.76 / 3.2 \mathrm{~mL}$, which was significantly lower than $6.02 / 3.2 \mathrm{~mL}$ in patients with stage III-IV tumors ( $p=0.049$ ) (Fig. 3d). The CTC subtype numbers from patients in the distant metastasis group were not significantly different from those without distant 
metastasis ( $p=0.180$ ) (Fig. 3e). However, total CTC number was markedly different between patients with and without distant metastasis ( $p=0.012$ ) (Fig. 3f).

Diagnostic value of CTC compared to CA199 in PDAC

We constructed ROC curves to assess the performance of CTCs as a diagnostic tool for PDAC (Fig. 4a). The value of the area under the ROC curve was up to $0.791(95 \% \mathrm{CI}=$ $0.718-0.863, p<0.001$ ) for total CTC number and 0.743 (95\% CI $=0.661-0.825, p<0.001$ ) for CTC subtype number. Youden's index was then used to select the optimum cutoff value which maximise the sensitivity and specificity from the ROC curve. The optimal cutoff value was 2 CTCs/3.2 mL whole blood for total CTC number, yielding a sensitivity of $75.8 \%$ and specificity of $68.7 \%$. For CTC subtype number, sensitivity was $53.7 \%$ and specificity was $85.4 \%$ using 2 CTC subtypes as the cutoff point. We defined samples with $\geq 2$ CTCs per $3.2 \mathrm{~mL}$ blood as CTC number-positive and those with $\geq 2$ CTC subtypes as CTC subtype-positive. The relationships between CTC and clinicopathological factors of PDAC were further examined (Table 2). Total CTC number-positive rates were not significantly associated with gender, age, tumor location, tumor differentiation, tumor size, tumor stage, lymph node metastasis, and metastasis, but showed a bias towards association with age. CTC subtype-positive rates were significantly associated with age and tumor location.

To further evaluate the diagnostic value of CTCs for PDAC screening, sensitivity and specificity of CA199 were compared with those of CTCs. ROC curve of CA199 was shown in Fig. 4b. The area under ROC curves was 0.831 (Table 3). Sensitivity and specificity were 0.684 and 0.979 for CA199. Compared with CA199, the diagnostic value of both total CTC number and CTC subtype number was a little poorer than CA199.

\section{Discussion}

CTCs are cancer cells disseminated from primary and metastatic tumors into the circulating system. They have been demonstrated to open a new research field in diagnosing, evaluating clinical effect, and monitoring recurrence in ovarian cancer [15]. Functional analysis of CTCs based on in vitro and in vivo models from breast, prostate and colon cancers have also attracted some interest due to the possibility of revealing the biological properties of metastatic cells [16]. The epithelial and mesenchymal markers of CTCs have been reported to change with disease progression in breast cancer [17]. Using innovative technology, CTCs could potentially aid in elucidating the molecular mechanisms of PDAC metastasis and chemoresistance, and assist in identifying therapeutic targets and determining follow-up treatment [18].

In our study, hypotonic hemolysis buffer was used to lyse RBCs and immunomagnetic beads used to remove the majority of leukocytes, leaving CTCs and a small quantity of leukocytes in the cell suspension. CD45, DAPI and CEP8-FISH staining data were combined to identify CTCs. Fluorescence in situ hybridization (FISH) techniques based on centromere probes have been utilized in particular chromosome aneuploidy detection. In an earlier study, sensitivity and specificity of FISH in aneuploidy detection were determined as $78 \%$ and $98 \%$ in 210 consecutive tissue samples from lung cancer patients [19]. CEP8-FISH has additionally been applied for CTC detection. Zhang et al. identified CTCs in 22 pancreatic cancers using a combination of CEP8, CK and CD45. Cells with features of CD45-/CEP8>2 were detected in 18 out of $22(81.8 \%)$ cancers [20]. Ning and co-workers combined immunostaining of CD45 and CEP8-FISH for detection of CTCs in lung cancers and ovarian cancers [12].The positive rate of lung cancer CTC detection was $83.33 \%$ (25/30). This method yielded a sensitivity of $76.2 \%(16 / 21)$ in ovarian cancers. In our experiments, CTCs were detected in 83 out of 95 (87.4\%) PDAC patients. These results support the utility of the method based on CD45, DAPI and CEP8-FISH staining for CTC identification.

The range of CTCs found in this study was 0-31 in PDAC patients and 0-5 in healthy controls, higher than that described in other studies. The difference in CTC number may result 
from the difference in CTC detection method. An efficient detection of CTCs is composed of effective isolation and accurate identification [21]. Several isolation methods have been used in pancreatic cancer patients, such as density centrifugation, immunuomagetic enrichment with the epithelial cell adhesion molecule (EpCAM) and cell filtration technology. CTC identification has been performed using CellSearch, immunocytochemistry and polymerase chain reaction (PCR) based method. Allard et al. used the CellSearch system to detect CTC in a total of 16 patients with metastatic pancreatic cancer [22]. 6 out of 16 (37.5\%) patients had CTCs, and only one patient had $\geq 5$ CTCs. Bidard et al. found CTCs in merely 9 out of 79 (11\%) patients with locally advanced pancreatic cancers using the same system [23]. Among them, six patients had $1 \mathrm{CTC} / 7.5 \mathrm{~mL}$, two patients had $2 \mathrm{CTC} / 7.5 \mathrm{~mL}$ and one patient had a high count of $15 \mathrm{CTC} / 7.5 \mathrm{~mL}$. Ren et al. identified CTC by immunocytochemistry via CA19-9 and cytokeratin $8 / 18$ and found that $33 / 41(80.5 \%)$ patients were CTC positive [24]. CTCs were detected in 33.8\%-84\% patients by Nested PCR using CK20 mRNA as CTC marker in different investigations $[25,26]$.

Here, we have reported for the first time that both CTC subtype number and total CTC number are upregulated in the peripheral blood of PDAC patients, compared with healthy controls. Total CTC number has been reported to be of value in pancreatic cancer diagnosis based on the CEP8-FISH method. Our findings imply that the CTC subtype number is also beneficial for tumor diagnosis. The current study has revealed novel insights into the potential diagnostic application of CTCs based on aneuploidy detection. Despite the increase in total CTC number in patients with distant metastasis relative to that in patients without metastasis, no association was evident between CTCs and metastasis. The CTCs detected in our study were actually circulating aneuploidies, reported to be a main driving force for tumorigenesis [27]. Few studies to date have reported a close relationship between aneuploidy and tumor metastasis. CTCs identified based on the EpCAM staining detection method were closely related to epithelial-mesenchymal transition (EMT) [28] and served as an independent prognostic factor for tumor metastasis [29]. The relationship between CTCs and tumor metastasis should be assessed based on the detection method.

Our study has several limitations. Total CTC number displayed a bias towards association with age and CTC subtype number was significantly associated with age. These results consisted with previous studies on cell and animal level. The accumulation of aneuploidies has been found in senescent endothelial cells [30]. Links between chromosomal instability and aging have been reported in mouse models [31,32]. Aneuploidy in the liver has also been reported to increase with age [33]. To improve the diagnostic application of CTC, it is necessary to remove disturbance from age-related aneuploidy. Notably, CTC subtypepositive rates were significantly associated with tumor location. 20/29 (68.97\%) patients with cancer in the pancreatic body or tail were CTC subtype-positive while merely 31/66 $(46.97 \%)$ patients with cancer in the pancreatic head or neck were CTC subtype-positive. This may be caused by the different serving vessels for different locations of pancreatic cancer [34]. Vascular invasion from the pancreatic head or neck cancer generally infiltrates the portal/superior mesenteric vein. Inversely, vascular invasion from the pancreatic body or tail cancer often occurs in the celiac arterial systems [35]. The different hematogenous transfer pathways of tumor cells from different locations may partly explain the different CTC subtype-positive rates in peripheral blood. Further studies are required to explain our findings. In view of the limitations in study time, the correlation of CTC number and subtype with prognosis needs to be further followed up over a longer period. The term 'CTCs' in our study actually refers to aneuploid cells in peripheral blood. The biological mechanisms of aneuploidy in association with tumor genesis and development require elucidation.

In summary, our data support the potential clinical value of PDAC CTCs based on a detection method combining CD45, DAPI and CEP8-FISH staining. Both CTC subtype number and total CTC number may act as potential biomarkers for PDAC diagnosis. The relationship between CTCs and patient prognosis will be further examined. Additional in-depth studies are required to elucidate the role of CTCs in PDAC progression. 


\section{Cellular Physiology Cell Physiol Biochem 2017;42:373-382 \\ \begin{tabular}{l|l|l} 
DOI: 10.1159/000477481 & O 2017 The Author(s). Published by S. Karger AG, Basel \\
www.karger.com/cpb
\end{tabular}}

Liu et al.: CTCs as a Biomarker in PDAC

\section{Acknowledgments}

This study was supported by a grant from the Scientific Research Foundation of Huashan Hospital (Grant No. 2016QD10).

\section{Disclosure Statement}

The authors have no conflict of interest.

\section{References}

1 Kamisawa T, Wood LD, Itoi T, Takaori K: Pancreatic cancer. Lancet 2016;388:73-85.

2 Al-Hawary M: Role of imaging in diagnosing and staging pancreatic cancer. J Natl Compr Canc Netw 2016;14:678-680.

-3 Khorana AA, Mangu PB, Berlin J, Engebretson A, Hong TS, Maitra A, Mohile SG, Mumber M, Schulick R, Shapiro M, Urba S, Zeh HJ, Katz MH: Potentially curable pancreatic cancer: American society of clinical oncology clinical practice guideline. J Clin Oncol 2016;34:2541-2556.

4 Ferrone CR, Finkelstein DM, Thayer SP, Muzikansky A, Fernandez-delCastillo C, Warshaw AL: Perioperative CA19-9 levels can predict stage and survival in patients with resectable pancreatic adenocarcinoma. J Clin Oncol 2006;24:2897-2902.

5 Nagrath S, Jack RM, Sahai V, Simeone DM: Opportunities and challenges for pancreatic circulating tumor cells. Gastroenterology 2016;151:412-426.

-6 Yu C, Wang Z, Xu X, Xiang W, Huang X: Circulating hepatocellular carcinoma cells are characterized by CXCR4 and MMP26. Cell Physiol Biochem 2015;36:2393-2402.

7 Fan JL, Yang YF, Yuan CH, Chen H, Wang FB: Circulating tumor cells for predicting the prognostic of patients with hepatocellular carcinoma: A meta analysis. Cell Physiol Biochem 2015;37:629-640.

8 Deneve E, Riethdorf S, Ramos J, Nocca D, Coffy A, Daures JP, Maudelonde T, Fabre JM, Pantel K, AlixPanabieres C: Capture of viable circulating tumor cells in the liver of colorectal cancer patients. Clin Chem 2013;59:1384-1392.

9 Kallergi G, Politaki E, Alkahtani S, Stournaras C, Georgoulias V: Evaluation of isolation methods for circulating tumor cells (CTCs). Cell physiol biochem 2016;40:411-419.

10 Danielsen HE, Pradhan M, Novelli M: Revisiting tumour aneuploidy - the place of ploidy assessment in the molecular era. Nat Rev Clin Oncol 2016;13:291-304.

-11 Pecot CV, Bischoff FZ, Mayer JA, Wong KL, Pham T, Bottsford-Miller J, Stone RL, Lin YG, Jaladurgam P, Roh JW, Goodman BW, Merritt WM, Pircher TJ, Mikolajczyk SD, Nick AM, Celestino J, Eng C, Ellis LM, Deavers MT, Sood AK: A novel platform for detection of CK+ and CK- CTCs. Cancer Discov 2011;1:580-586.

-12 Ning N, Zhan T, Zhang Y, Chen Q, Feng F, Yang Z, Liu Z, Xu D, Wang F, Guo Y, Xing J, Guan Y, Cui W: Improvement of specific detection of circulating tumor cells using combined CD45 staining and fluorescence in situ hybridization. Clin Chim Acta 2014;433:69-75.

13 Biesterfeld S, Gerres K, Fischer-Wein G, Bocking A: Polyploidy in non-neoplastic tissues. J Clin Pathol 1994;47:38-42.

14 Storchova Z, Pellman D: From polyploidy to aneuploidy, genome instability and cancer. Nat Rev Mol Cell Biol 2004;5:45-54.

15 Gasparri ML, Savone D, Besharat RA, Farooqi AA, Bellati F, Ruscito I, Panici PB, Papadia A: Circulating tumor cells as trigger to hematogenous spreads and potential biomarkers to predict the prognosis in ovarian cancer. Tumour Biol 2016;37:71-75.

16 Pantel K, Alix-Panabieres C: Functional Studies on Viable Circulating Tumor Cells. Clin Chem 2016;62:328334. 


\section{Cellular Physiology Cell Physiol Biochem 2017;42:373-382

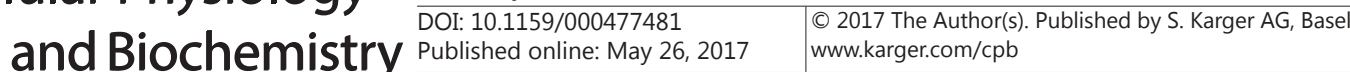

Liu et al.: CTCs as a Biomarker in PDAC

-17 Yu M, Bardia A, Wittner BS, Stott SL, Smas ME, Ting DT, Isakoff SJ, Ciciliano JC, Wells MN, Shah AM, Concannon KF, Donaldson MC, Sequist LV, Brachtel E, Sgroi D, Baselga J, Ramaswamy S, Toner M, Haber DA, Maheswaran S: Circulating breast tumor cells exhibit dynamic changes in epithelial and mesenchymal composition. Science 2013;339:580-584.

18 Cen P, Ni X, Yang J, Graham DY, Li M: Circulating tumor cells in the diagnosis and management of pancreatic cancer. Biochim Biophys Acta 2012;1826:350-356.

19 Schramm M, Wrobel C, Born I, Kazimirek M, Pomjanski N, William M, Kappes R, Gerharz CD, Biesterfeld S, Bocking A: Equivocal cytology in lung cancer diagnosis: Improvement of diagnostic accuracy using adjuvant multicolor FISH, DNA-image cytometry, and quantitative promoter hypermethylation analysis. Cancer Cytopathol 2011;119:177-192.

20 Zhang Y, Wang F, Ning N, Chen Q, Yang Z, Guo Y, Xu D, Zhang D, Zhan T, Cui W: Patterns of circulating tumor cells identified by CEP8, CK and CD45 in pancreatic cancer. Int J Cancer 2015;136:1228-1233.

21 Tjensvoll K, Nordgard 0, Smaaland R: Circulating tumor cells in pancreatic cancer patients: Methods of detection and clinical implications. Int J Cancer 2014;134:1-8.

22 Allard WJ, Matera J, Miller MC, Repollet M, Connelly MC, Rao C, Tibbe AG, Uhr JW, Terstappen LW: Tumor cells circulate in the peripheral blood of all major carcinomas but not in healthy subjects or patients with nonmalignant diseases. Clin Cancer Res 2004;10:6897-6904.

23 Bidard FC, Huguet F, Louvet C, Mineur L, Bouche O, Chibaudel B, Artru P, Desseigne F, Bachet JB, Mathiot C, Pierga JY, Hammel P: Circulating tumor cells in locally advanced pancreatic adenocarcinoma: The ancillary CirCe 07 study to the LAP 07 trial. Ann Oncol 2013;24:2057-2061.

24 Ren C, Han C, Zhang J, He P, Wang D, Wang B, Zhao P, Zhao X: Detection of apoptotic circulating tumor cells in advanced pancreatic cancer following 5-fluorouracil chemotherapy. Cancer Biol Ther 2011;12:700-706.

25 Zhou J, Hu L, Yu Z, Zheng J, Yang D, Bouvet M, Hoffman RM: Marker expression in circulating cancer cells of pancreatic cancer patients. J Surg Res 2011;171:631-636.

-26 Soeth E, Grigoleit U, Moellmann B, Roder C, Schniewind B, Kremer B, Kalthoff H, Vogel I: Detection of tumor cell dissemination in pancreatic ductal carcinoma patients by CK 20 RT-PCR indicates poor survival. J Cancer Res Clin Oncol 2005;131:669-676.

27 Davoli T, Xu AW, Mengwasser KE, Sack LM, Yoon JC, Park PJ, Elledge SJ: Cumulative haploinsufficiency and triplosensitivity drive aneuploidy patterns and shape the cancer genome. Cell 2013;155:948-962.

28 Mohamadi RM, Besant JD, Mepham A, Green B, Mahmoudian L, Gibbs T, Ivanov I, Malvea A, Stojcic J, Allan AL, Lowes LE, Sargent EH, Nam RK, Kelley SO: Nanoparticle-mediated binning and profiling of heterogeneous circulating tumor cell subpopulations. Angew Chem Int Ed Engl 2015;54:139-143.

29 Bidard FC, Mathiot C, Delaloge S, Brain E, Giachetti S, de Cremoux P, Marty M, Pierga JY: Single circulating tumor cell detection and overall survival in nonmetastatic breast cancer. Ann Oncol 2010;21:729-733.

-30 Wagner M, Hampel B, Bernhard D, Hala M, Zwerschke W, Jansen-Durr P: Replicative senescence of human endothelial cells in vitro involves G1 arrest, polyploidization and senescence-associated apoptosis. Exp Gerontol 2001;36:1327-1347.

-31 Baker DJ, Jeganathan KB, Cameron JD, Thompson M, Juneja S, Kopecka A, Kumar R, Jenkins RB, de Groen PC, Roche P, van Deursen JM: BubR1 insufficiency causes early onset of aging-associated phenotypes and infertility in mice. Nat Genet 2004;36:744-749.

-32 Wijshake T, Malureanu LA, Baker DJ, Jeganathan KB, van de Sluis B, van Deursen JM: Reduced life- and healthspan in mice carrying a mono-allelic BubR1 MVA mutation. Plos Genet 2012;8:e1003138.

-33 Kudryavtsev BN, Kudryavtseva MV, Sakuta GA, Stein GI: Human hepatocyte polyploidization kinetics in the course of life cycle. Virchows Arch B Cell Pathol Incl Mol Pathol 1993;64:387-393.

-34 Kanda M, Fujii T, Sahin TT, Kanzaki A, Nagai S, Yamada S, Sugimoto H, Nomoto S, Takeda S, Kodera Y, Morita S, Nakao A: Invasion of the splenic artery is a crucial prognostic factor in carcinoma of the body and tail of the pancreas. Ann Surg 2010;251:483-487.

-35 Hirano S, Kondo S, Hara T, Ambo Y, Tanaka E, Shichinohe T, Suzuki O, Hazama K: Distal pancreatectomy with en bloc celiac axis resection for locally advanced pancreatic body cancer: Long-term results. Ann Surg 2007;246:46-51. 